\title{
Modeling and Numerical Simulation for the Kinematics of Six Revolute Joint Robot
}

\author{
Baolin Yin ${ }^{a}$, Bingbing Yan ${ }^{b}$, Yushuo Wang ${ }^{c}$ and Xiaoming Feng ${ }^{d}$ \\ School of Mechanical Engineering, Jiamusi University, Jiamusi 154007, China \\ ayinblin@163.com, byanbingbing@126.com, cwys1459@163.com, d1159527506@qq.com \\ Corresponding author: Bingbing Yan
}

Keywords: Robot, direct kinematics, inverses kinematics, D-H method.

\begin{abstract}
The establishment of the kinematics model for robot, including of direct kinematics and inverses kinematics, which is the base of the application of the robot. This paper presents the kinematics model of the six revolute joint robot. Through analysis position with D-H method, the direct kinematics model and inverse kinematics model are established. Given the geometric parameters of the six revolute joint robot, the numerical examples are provided. Therefore, the direct kinematics model and inversed kinematics model are minimal. And the research is applicable to other robot systems with similar mechanical configurations.
\end{abstract}

\section{Introduction}

It is important to establish the kinematics model for revolute joint robot, which is the base of the application of the robot [1]. The models include direct kinematics and inverses kinematics. One of the challenges in studying robot consists of the difficulty in solving their inverse kinematics problems, which leads to systems of polynomial equations. And solution approaches for such a problem can be divided into two classes: numerical methods and analytic techniques [2]. Denavit proposed the method, which presents the pose relation of links with displacement transformation matrix, named $\mathrm{D}-\mathrm{H}$ method [3]. Then the D-H method is applied in establishment of the kinematics model for robot. The inverses kinematics model for PUMA 560 robot was presented [4]. The equations of inverses kinematics were derived for MOTOMAN-UPJ [5, 6]. In [7], the kinematics model was established for the six revolute joint robot, named 6R robot. And the Graphic simulation was provided.

It is important to obtain the dominant type of the kinematics model. In addition, extraneous solution is provided through deriving equations, results from trigonometric function transformation. In [8], the inverses kinematics was analyzed, and sixteen real solutions were obtained. In this paper, the kinematics model for $6 \mathrm{R}$ robot is present with $\mathrm{D}-\mathrm{H}$ method, and the numerical examples are provided.

\section{Direct Kinematics for $6 \mathrm{R}$ robot}

\section{Direct Kinematics Model.}

Fig. 1 shows the schematic of the D-H method. Link $i-1$ is connected with link $i$ by joint $i$. A reference frame $O_{i \text {-xiyizi }}$ attached to the joint $i$ is defined as: $z_{i}$ axes and joint $i$ axes coincide, and the $x_{i}$ axes is perpendicular to the $z_{i}$ axes and $z_{i+1}$ axes. Joint $i$ axes defined as axis of rotation for revolute, or the direction of movement for the prismatic pair. Here, torsion angle $\alpha_{i}$ is defined from $z_{i-1}$ axes to $z_{i}$ axes, revolute angle $\theta_{i}$ is defined from $x_{i-1}$ axes to $x_{i}$ axes, $a_{i}$ is defined as length of link $i$, di is defined as offset distance from $\mathrm{x}_{i}$ to $x_{i+1}$. The pose relation of link $i-1$ and link $i$ can be given with displacement transformation matrix by $\mathrm{D}-\mathrm{H}$ method, as 


$$
{ }_{i}^{i-1} T=\left[\begin{array}{cccc}
c_{i} & -s_{i} & 0 & a_{i-1} \\
s_{i} \cos \alpha_{i-1} & c_{i} \cos \alpha_{i-1} & -\sin \alpha_{i-1} & -d_{i} \sin \alpha_{i-1} \\
s_{i} \sin \alpha_{i-1} & c_{i} \sin \alpha_{i-1} & \cos \alpha_{i-1} & d_{i} \cos \alpha_{i-1} \\
0 & 0 & 0 & 1
\end{array}\right] \text {. }
$$

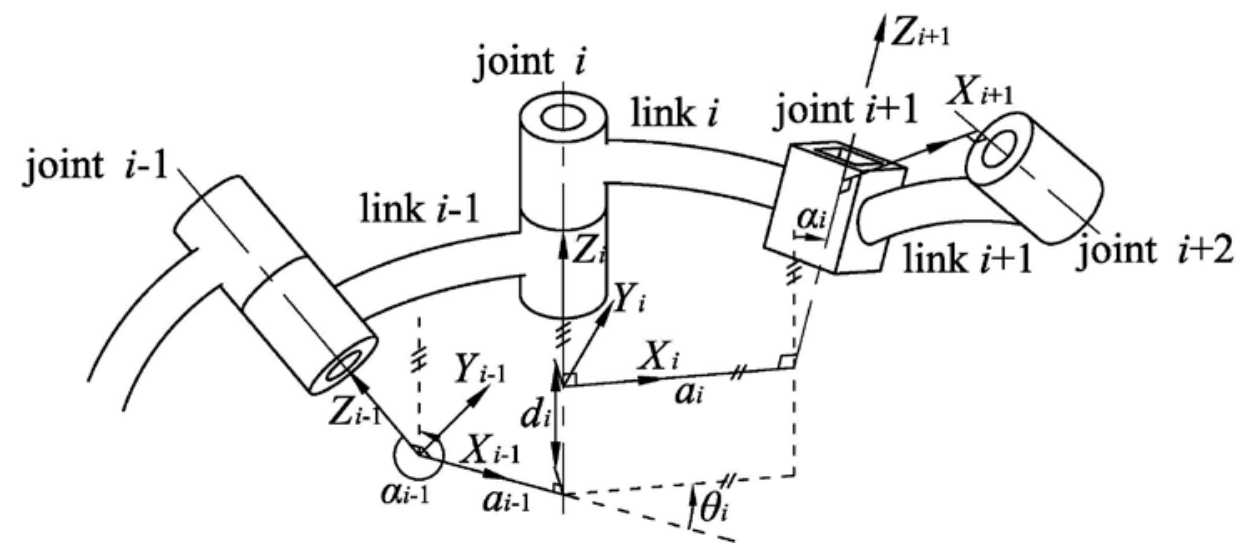

Fig. 1 Schematic of the D-H method

Where $s_{i}=\sin \left(\theta_{i}\right), \mathrm{c}_{i}=\cos \left(\theta_{i}\right)$.

For 6R robot, the displacement transformation matrix for inertial coordinate system to end effecter coordinate system can be given as

$$
{ }_{6}^{0} T={ }_{1}^{0} T{ }_{2}^{1} T{ }_{3}^{2} T{ }_{4}^{3} T{ }_{5}^{4} T{ }_{6}^{5} T \text {. }
$$

Substituting Eq. 1 into Eq. 2, yields

$$
{ }_{6}^{0} T=\left[\begin{array}{cccc}
t_{11} & t_{12} & t_{13} & t_{14} \\
t_{21} & t_{22} & t_{23} & t_{24} \\
t_{31} & t_{32} & t_{33} & t_{34} \\
0 & 0 & 0 & 1
\end{array}\right] .
$$

For 6R robot in this paper, the link parameters are given as Table 1 . The reference coordinate systems are shown as Fig. 2. And then, substituting the parameters into Eq.1, Eq.2 and Eq.3, where $t_{i j}$ can be expressed as

Table 1 Link parameters for 6R robot

\begin{tabular}{ccccc}
\hline i & ai-1 & $\alpha \mathrm{i}-1$ & $\theta \mathrm{i}$ & $\mathrm{di}$ \\
\hline 1 & 0 & 0 & $-180 \mathrm{o} \sim 180 \mathrm{o}$ & 0 \\
2 & $\mathrm{a} 1$ & $-90 \mathrm{o}$ & $-170 \mathrm{o} \sim-90 \mathrm{o}$ & 0 \\
3 & $\mathrm{a} 2$ & 0 & $225 \mathrm{o} \sim 70 \mathrm{o}$ & 0 \\
4 & $\mathrm{a} 3$ & $-90 \mathrm{o}$ & $-180 \mathrm{o} \sim 180 \mathrm{o}$ & $\mathrm{d} 4$ \\
5 & 0 & $90 \mathrm{o}$ & $-150 \mathrm{o} \sim 150 \mathrm{o}$ & 0 \\
6 & 0 & $-90 \mathrm{o}$ & $-180 \mathrm{o} \sim 180 \mathrm{o}$ & 0 \\
\hline
\end{tabular}




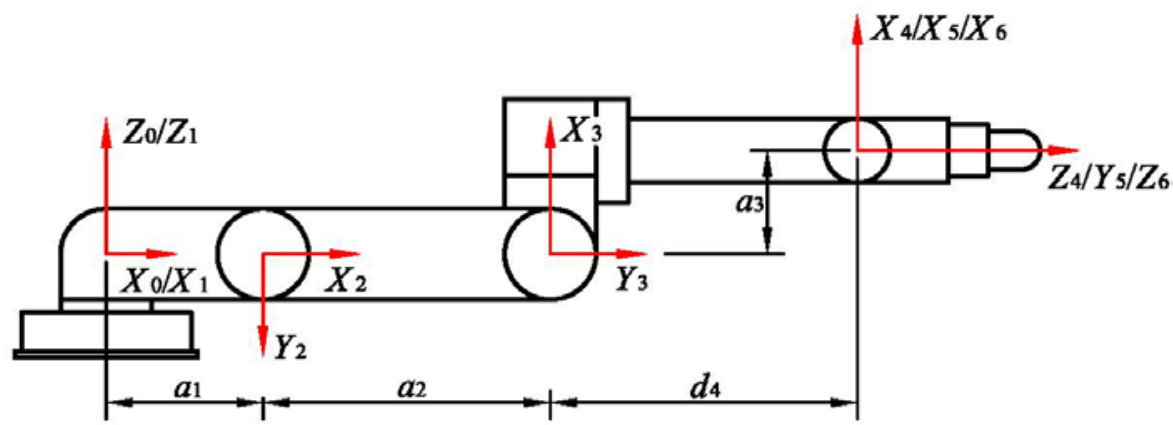

Fig. 2 Schematic of the reference coordinate systems

$t_{11}=\left(\left(\left(c_{1} c_{2} c_{3}-c_{1} S_{2} S_{3}\right) c_{4}+s_{1} S_{4}\right) c_{5}+\left(-c_{1} c_{2} S_{3}-c_{1} S_{2} c_{3}\right) s_{5}\right) c_{6}-\left(\left(c_{1} c_{2} c_{3}-c_{1} S_{2} S_{3}\right) s_{4}-s_{1} c_{4}\right) s_{6}$

$t_{12}=-\left(\left(\left(c_{1} c_{2} c_{3}-c_{1} S_{2} s_{3}\right) c_{4}+s_{1} s_{4}\right) c_{5}+\left(-c_{1} C_{2} s_{3}-c_{1} S_{2} c_{3}\right) s_{5}\right) s_{6}-\left(\left(c_{1} c_{2} c_{3}-c_{1} S_{2} S_{3}\right) s_{4}-s_{1} c_{4}\right) c_{6}$

$t_{13}=-\left(\left(c_{1} C_{2} c_{3}-c_{1} S_{2} s_{3}\right) c_{4}+s_{1} s_{4}\right) s_{5}+\left(-c_{1} c_{2} S_{3}-c_{1} s_{2} c_{3}\right) c_{5}$

$t_{14}=c_{1} a_{1}+c_{1} c_{2} a_{2}+\left(c_{1} c_{2} c_{3}-c_{1} s_{2} s_{3}\right) a_{3}+\left(-c_{1} c_{2} S_{3}-c_{1} S_{2} c_{3}\right) d_{4}$

$t_{21}=\left(\left(\left(s_{1} C_{2} c_{3}-S_{1} S_{2} S_{3}\right) c_{4}-c_{1} S_{4}\right) c_{5}+\left(-s_{1} C_{2} S_{3}-S_{1} S_{2} C_{3}\right) s_{5}\right) c_{6}-\left(\left(s_{1} C_{2} C_{3}-S_{1} S_{2} S_{3}\right) s_{4}+c_{1} S_{4}\right) s_{6}$

$t_{22}=-\left(\left(\left(s_{1} c_{2} c_{3}-s_{1} S_{2} S_{3}\right) c_{4}-c_{1} S_{4}\right) c_{5}+\left(-s_{1} c_{2} S_{3}-s_{1} S_{2} c_{3}\right) s_{5}\right) s_{6}-\left(\left(s_{1} C_{2} c_{3}-s_{1} S_{2} S_{3}\right) s_{4}+c_{1} c_{4}\right) c_{6}$

$t_{23}=-\left(\left(s_{1} c_{2} c_{3}-s_{1} s_{2} s_{3}\right) c_{4}-c_{1} s_{4}\right) s_{5}+\left(-s_{1} c_{2} S_{3}-s_{1} S_{2} c_{3}\right) c_{5}$

$t_{24}=s_{1} a_{1}+s_{1} c_{2} a_{2}+\left(s_{1} c_{2} c_{3}-s_{1} s_{2} s_{3}\right) a_{3}+\left(-s_{1} c_{2} s_{3}-s_{1} s_{2} c_{3}\right) d_{4}$

$t_{31}=\left(\left(-s_{2} c_{3}-c_{2} s_{3}\right) c_{4} c_{5}+\left(s_{2} s_{3}-c_{2} c_{3}\right) s_{5}\right) c_{6}-\left(-s_{2} c_{3}-c_{2} s_{3}\right) s_{4} s_{6}$

$t_{32}=-\left(\left(-S_{2} c_{3}-c_{2} S_{3}\right) c_{4} c_{5}+\left(s_{2} S_{3}-c_{2} c_{3}\right) s_{5}\right) s_{6}-\left(-s_{2} c_{3}-c_{2} s_{3}\right) s_{4} c_{6}$

$t_{33}=-\left(\left(-s_{2} c_{3}-c_{2} s_{3}\right) c_{4} s_{5}+\left(s_{2} s_{3}-c_{2} c_{3}\right) c_{5}\right) c_{5}$

$t_{34}=-s_{2} a_{2}+\left(-s_{2} c_{3}-c_{2} s_{3}\right) a_{3}+\left(s_{2} s_{3}-c_{2} c_{3}\right) d_{4}$

Here Z-Y-X type Euler angular is used to defined the orientation of the end effecter as $[\alpha \beta \gamma]^{\mathrm{T}}$, and the positon is defined as $P_{6}=\left[\begin{array}{lll}x_{P} & y_{P} Z_{P}\end{array}\right]^{\mathrm{T}}$, yields

$$
{ }_{6}^{0} T=\left[\begin{array}{cccc}
c \alpha c \beta & c \alpha s \beta s \gamma-s \alpha c \gamma & c \alpha s \beta c \gamma+s \alpha s \gamma & x_{\mathrm{p}} \\
s \alpha c \beta & s \alpha s \beta s \gamma+c \alpha c \gamma & s \alpha s \beta c \gamma-c \alpha s \gamma & y_{\mathrm{p}} \\
-s \beta & c \beta s \gamma & c \beta c \gamma & z_{\mathrm{p}} \\
0 & 0 & 0 & 1
\end{array}\right] \text {. }
$$

Form Eq.3, Eq.4 and Eq. 5, the direct kinematics model of 6R robot can been given as

$$
P O S=\left[\begin{array}{c}
\alpha \\
\beta \\
\gamma \\
x_{\mathrm{P}} \\
y_{\mathrm{P}} \\
z_{\mathrm{P}}
\end{array}\right]=\left[\begin{array}{c}
\arctan \left(t_{21} / t_{11}\right) \\
\arctan \left(-t_{31} / \sqrt{t_{11}^{2}+t_{21}^{2}}\right) \\
\arctan \left(t_{32} / t_{33}\right) \\
t_{14} \\
t_{24} \\
t_{34}
\end{array}\right] .
$$

Given driving parameters $\theta_{i}$, the $t_{i j}$ can be obtained from Eq.4, and the direct kinematics model of 6R robot express as Eq. 6 is expressed as dominant type.

Numerical Example for Direct Kinematics Model.

As an example of the direct kinematics solution for a general case mechanism, let the link parameters be as: $\mathrm{a} 1=150 \mathrm{~mm}, \mathrm{a} 2=250 \mathrm{~mm}, \mathrm{a} 3=60 \mathrm{~mm}, \mathrm{~d} 4=140 \mathrm{~mm}$. And the driving parameters $\theta \mathrm{i}$ is given as

$$
\left.\begin{array}{lll}
\theta_{1}=30-12 t, & \theta_{2}=-100-8 t, & \theta_{3}=-160+18 t \\
\theta_{4}=100+9 t, & \theta_{5}=110+7 t, & \theta_{6}=60+16 t,
\end{array}\right\} \quad 0 \leq t \leq 5 .
$$

The orientation and the positon of the end effecter, which are changed with value $t$ are shown in Fig. 3. So, given driving parameters $\theta \mathrm{i}$, the orientation and the positon of the end effecter can be obtained. Therefore, the direct kinematics model for the 6R robot is minimal. 


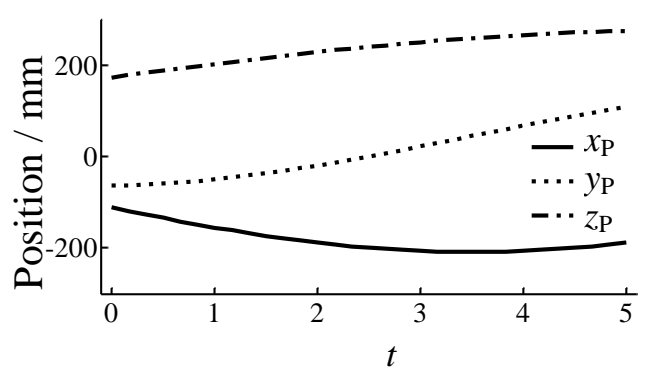

(a) Positon is changed with value $t$

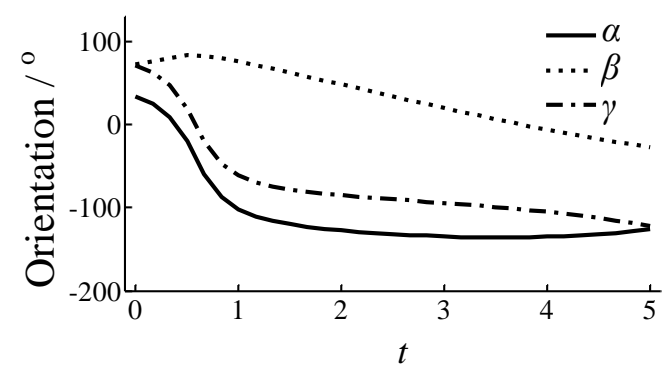

(b) Orientation is changed with value $t$

Fig. 3 Orientation and positon of the end effecter, which are changed with value $t$

\section{Inverses Kinematics for $6 \mathrm{R}$ robot}

\section{Inverses Kinematics model.}

According to end effecter vector POS, the solution of the corresponding driving parameters $\theta \mathrm{i}$ can be solved. This process is called inverse kinematics.

By matrix transform, yields

$$
{ }_{6}^{2} T={ }_{1}^{0} T^{-1}{ }_{6}^{0} T={ }_{2}^{1} T \quad{ }_{3}^{2} T{ }_{4}^{3} T \quad{ }_{5}^{4} T \quad{ }_{6}^{5} T \text {. }
$$

And from ${ }_{6}^{2} T(2,4)$, which can be derived as

$y_{\mathrm{p}} c_{1}-x_{\mathrm{p}} s_{1}=0$.

The half-angel tangent relationships is substituted, and the $\theta 1$ can been given as

$$
\theta_{1}=2 \arctan \left(\frac{-1 \pm \sqrt{1+\left(y_{\mathrm{p}} / x_{\mathrm{p}}\right)^{2}}}{\left(y_{\mathrm{p}} / x_{\mathrm{p}}\right)}\right) \text {. }
$$

By matrix transform, yields

${ }_{5}^{3} T={ }_{2}^{1} T^{-1}{ }_{1}^{0} T^{-1}{ }_{6}^{0} T{ }_{5}^{5} T^{-1}={ }_{3}^{2} T{ }_{4}^{3} T{ }_{5}^{4} T$.

And from ${ }_{5}^{3} T(1,4)$ and ${ }_{5}^{3} T(2,4)$, which can be derived as

$$
\begin{aligned}
& a_{3} c_{3}-d_{4} s_{3}+a_{2}=c_{2}\left(c_{1} x_{\mathrm{p}}+s_{1} y_{\mathrm{p}}-a_{1}\right)-s_{2} z_{\mathrm{p}} \\
& a_{3} s_{3}+d_{4} c_{3}=-s_{2}\left(c_{1} x_{\mathrm{p}}+s_{1} y_{\mathrm{p}}-a_{1}\right)-c_{2} z_{\mathrm{p}}
\end{aligned} .
$$

Then the $\theta 2$ and $\theta 3$ can been given as

$$
\begin{aligned}
& \theta_{2}=2 \arctan \left(\frac{-z_{\mathrm{p}} \pm \sqrt{z_{\mathrm{p}}^{2}+u^{2}-v^{2}}}{u-v}\right) \\
& \theta_{3}=2 \arctan \left(\frac{-a_{3} \pm \sqrt{a_{3}^{2}+d_{4}^{2}-w^{2}}}{w-d_{4}}\right) .
\end{aligned}
$$

Where $\mathrm{u}, \mathrm{v}$ and $\mathrm{w}$ can be expressed as

$$
\begin{aligned}
& u=x_{\mathrm{p}} c_{1}+y_{\mathrm{p}} s_{1}-a_{1} \\
& v=\frac{-a_{2}^{2}+a_{3}^{2}+d_{4}^{2}-u^{2}-z_{\mathrm{p}}^{2}}{2 a_{2}} . \\
& w=u s_{2}+z_{\mathrm{p}} c_{2}
\end{aligned}
$$

By matrix transform, yields

$$
{ }_{6}^{3} T={ }_{3}^{2} T^{-1}{ }_{2}^{1} T^{-1}{ }_{1}^{0} T^{-1}{ }_{6}^{0} T={ }_{4}^{3} T \quad{ }_{5}^{4} T{ }_{6}^{6} T \text {. }
$$

From ${ }_{6}^{3} T(2,3),{ }_{6}^{3} T(1,3)$ and ${ }_{6}^{3} T(2,1)$, which can be derived as 


$$
\begin{aligned}
& c_{5}=\eta \\
& -c_{4} S_{5}=\lambda . \\
& S_{5} C_{6}=\mu
\end{aligned}
$$

Where $\eta, \lambda$ and $\mu$ can be expressed as

$$
\begin{aligned}
& \eta=c_{1}\left(-c_{2} s_{3}-s_{2} c_{3}\right) t_{13}+s_{1}\left(-c_{2} s_{3}-s_{2} c_{3}\right) t_{23}+\left(-c_{2} c_{3}+s_{2} s_{3}\right) t_{33} \\
& \lambda=c_{1}\left(c_{2} c_{3}-s_{2} s_{3}\right) t_{13}+s_{1}\left(c_{2} c_{3}-s_{2} s_{3}\right) t_{23}+\left(-c_{2} s_{3}-s_{2} c_{3}\right) t_{33} \\
& \mu=c_{1}\left(-c_{2} s_{3}-s_{2} c_{3}\right) t_{11}+s_{1}\left(-c_{2} s_{3}-s_{2} c_{3}\right) t_{21}+\left(-c_{2} c_{3}+s_{2} s_{3}\right) t_{31}
\end{aligned} .
$$

Then the $\theta 4, \theta 5$ and $\theta 6$ can been given as

$$
\begin{aligned}
& \theta_{5}=2 \arctan \left( \pm \sqrt{\frac{1-\eta}{1+\eta}}\right) \\
& \theta_{4}=2 \arctan \left( \pm \sqrt{\frac{1+\lambda / s_{5}}{1-\lambda / s_{5}}}\right) . \\
& \theta_{6}=2 \arctan \left( \pm \sqrt{\frac{1-\mu / s_{5}}{1+\mu / s_{5}}}\right)
\end{aligned}
$$

Given the end effecter vector POS0, the $\mathrm{u}, \mathrm{v}, \mathrm{w}, \eta, \lambda$ and $\mu$ can be obtained from Eq.14 and Eq.17, and driving parameters $\theta \mathrm{i}$ can be solved from Eq. 10, Eq. 13 and Eq. 18. The inverses kinematics model of 6R robot is expressed as dominant type.

From the inverses kinematics model, the sixty-four real solutions can be obtained, which include the extraneous solution results from trigonometric function transformation. In this paper, driving parameters $\theta \mathrm{i}$ are substituted into Eq. 4 and Eq. 6, and the vector POSi can be obtained. And the discriminate of the real solutions for the inverses kinematics model can be given as

$$
\left\|P O S_{i}-P_{0}\right\| \leq 1 \times 10^{-5} \text {. }
$$

\section{Numerical Example for Inverses Kinematics model.}

As an example of the inverses kinematics solution, let the driving parameters $\theta=\left[-20^{\circ},-120^{\circ}, 100^{\circ}\right.$, $\left.60^{\circ}, 30^{\circ}, 40^{\circ}\right]^{\mathrm{T}}$, and then POS0 $=\left[64.8724^{\circ}, 25.9040^{\circ}, 1.2795^{\circ}, 121.4688,-44.2110,105.4706\right]^{\mathrm{T}}$. The roots for $\theta_{i}$ are given in Table 2 . There are eight real roots. So, for the given $P_{0}$, there are eight groups driving parameters for the $6 \mathrm{R}$ robot. Those real roots are depicted in Fig. 4 . Therefore, the direct kinematics is minimal.

Table 2 Solutions of $\theta \mathrm{i}$ for the inverses kinematics

\begin{tabular}{ccccccc}
\hline No. & $\theta_{1} /^{0}$ & $\theta_{2} /^{0}$ & $\theta_{3} /^{0}$ & $\theta_{4} /{ }^{\circ}$ & $\theta_{5} /^{0}$ & $\theta_{6} /{ }^{\circ}$ \\
\hline 1 & -20 & -82.2451 & 126.3972 & 147.1410 & 52.9464 & -62.4239 \\
2 & -20 & -82.2451 & 126.3972 & -32.8590 & -52.9464 & 117.5761 \\
3 & -20 & -120 & 100 & 60 & 30 & 40 \\
4 & -20 & -120 & 100 & -120 & -30 & -140 \\
5 & 160 & -128.6640 & -154.2331 & -153.3530 & 74.9028 & 88.8639 \\
6 & 160 & -128.6640 & -154.2331 & 26.6470 & -74.9028 & -91.1361 \\
7 & 160 & 170.0445 & 20.63030 & -76.1988 & 153.5200 & -158.344 \\
8 & 160 & 170.0445 & 20.63030 & 103.8012 & -153.5200 & 21.6562 \\
\hline
\end{tabular}




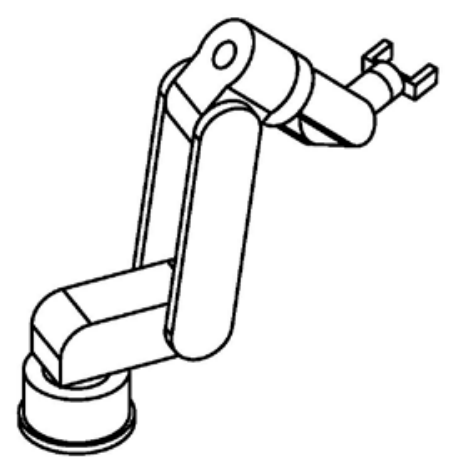

(1) No.1

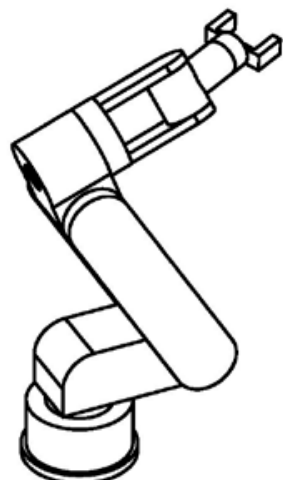

(b) No.3

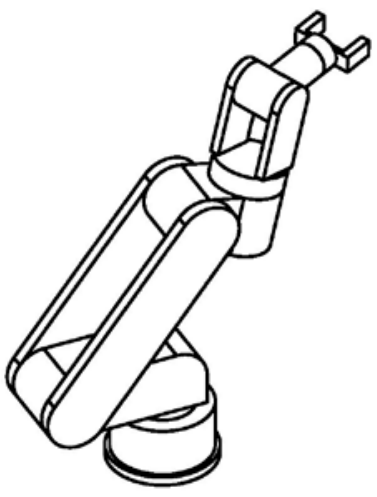

(c) No.5

Fig. 4 Schematic of the inverses kinematics

\section{Summary}

(1) Based on D-H method, the direct kinematics model for the 6R robot has been successfully approached. And the model is expressed as dominant type. Then numerical example is included. Therefore, the direct kinematics model for the 6R robot is minimal.

(2) Based on D-H method, the inverses kinematics model for the 6R robot has been successfully approached. And the model is expressed as dominant type. The discriminate of the real solutions for the model are obtained. Then numerical example is included, there are eight real roots. Therefore, the inverses kinematics model for the $6 \mathrm{R}$ robot is minimal.

\section{Acknowledgments}

This work has been supported by science and technology research projects of Heilongjiang Province Office of Education (Item Number 12531671), the Innovation and Entrepreneurship Fund of Jiamusi University (xzyf2013-02) and the New Century Excellent Talents in Heilongjiang Provincial University (1252-NCET-021).

\section{References}

[1]. P.PAUL, BRUCE SHIMANO, GORDON E. IEEE Transactions on Systems, Man and Cybernetics, 1981, p. 449-455.

[2]. J. E. Shigley. In. Kinematic Analysis of Mechanisms. New York, McGraw-Hill, 1959.

[3]. J. Denavit, R. S. Hartenberg. Journal of Applied Mechanics. Vol. 22(1955), p. 215-221.

[4]. W. Qizh, X. Xinhe, Y. Chaowan. Robot. Vol. 20 (1998), p. 81-87.

[5]. W. Xuesong, X. Shifan, H. Jifei. Journal of China University of Mining \& Technology. Vol. 30 (2001), p. 73-75.

[6]. C. Ping, L. Guohai. Journal of Mechanical Transmission. Vol. 30 (2006), p. 23-27.

[7]. W. Liquan, L. Bingyi, W. Janrong, H. Jinhua, L. Zhengyu. CAAI Transactions on Intelligent Systems. Vol. 5 (2010), p. 156-160.

[8]. L. Yajun, H. Tian. Journal of Mechanical Engineering. Vol. 48 (2012), p. 9-15. 